\title{
Fast single loop diamagnetic measurements on the TCV Tokamak
}

\author{
J.-M. Moret, F. Buhlmann ${ }^{1}$, G. Tonetti \\ CRPP - EPFL \\ Centre de Recherches en Physique des Plasmas \\ Association EURATOM - Confédération Suisse \\ École Polytechnique Fédérale de Lausanne \\ CH-1015, Lausanne, Switzerland
}

Measurement of the plasma generated diamagnetic flux on TCV is used to derive the plasma pressure, as on other magnetic confinement experiments. However specificities of the device make the measurement more difficult: for passive stabilisation of the vertical position of highly elongated plasmas, the vessel has a low electrical resistivity, leading to large image currents in the vessel. For the same reason the plasma must also be kept close to the conducting wall, so that the in-vessel double loop method usually used to compensate for these currents can not be applied. In order to achieve proper compensation, the diamagnetic measurement diagnostic on TCV uses the signal from a single loop wound outside the vessel in combination with appropriate signal processing that accurately matches the fast component of the induced vessel current. This allows extraction of the plasma diamagnetic flux with a remarkable bandwidth, of $\sim 10 \mathrm{kHz}$, and with the required accurate compensation of $0.05 \mathrm{mWb}$ out of $2 \mathrm{~Wb}$. As a result, rapid changes in plasma pressure can be studied, for example during additional heating power modulation or plasma instabilities such as ELMs.

\section{Introduction}

TCV (Tokamak à configuration variable) was built to study the influence of the plasma shape on tokamak physics and plasma performance [1][2]. Equilibria include limited and open divertor configurations with an elongation up to 2.8, upper and lower single null or double null divertors and even more complex configurations such as doublets. The machine was designed to maintain flexible control of the exact contour of the plasma column and of the divertor leg geometry in all these configurations. It is also equipped with a powerful electron cyclotron resonance heating $(\mathrm{ECH})$ system [3][4] and the physics of this heating scheme and of the plasma

1. Present address: Compex SA, 1024 Ecublens, Switzerland 
regimes that are obtained using it constitute a major aspect of the experimental program [5]. Relevant parameters of the TCV tokamak are given in table 1.

\begin{tabular}{|l|l|l|}
\hline Major radius & $R_{0}$ & $0.90 \mathrm{~m}$ \\
\hline Horizontal minor radius & $a$ & $0.25 \mathrm{~m}$ \\
\hline Maximum elongation & $\kappa$ & 3 \\
\hline Maximum toroidal field & $B_{t}$ & $1.5 \mathrm{~T}$ \\
\hline Plasma current & $I_{p}$ & $0.1-1 \mathrm{MA}$ \\
\hline ECH heating power & & $4.5 \mathrm{MW}$ \\
\hline
\end{tabular}

Table 1: TCV parameters

Diamagnetic measurements are commonly used in fusion devices [6] to derive the normalised plasma pressure, or poloidal beta, $\beta_{p}$ from the toroidal magnetic flux produced by the plasma, $\phi_{p}$ using the equilibrium relation, given here in its simplified form

$$
\phi_{p}=\frac{\mu_{o}^{2} I_{p}^{2}}{8 \pi B_{t}}\left(1-\beta_{p}\right) .
$$

For typical TCV plasma parameters, this flux ranges from $0.4 \mathrm{mWb}$ at low $I_{p}$ to $40 \mathrm{mWb}$ at the highest plasma current. To derive $\beta_{p}$ with a reasonable confidence of $10 \%$, an absolute accuracy as low as $0.04 \mathrm{mWb}$ is required on the measurement, a value which, when compared to the flux of $2 \mathrm{~Wb}$ produced by the main toroidal field, corresponds to $20 \mathrm{ppm}$.

In addition to the measurement precision, one must also consider its bandwidth. The plasma energy confinement time, which gives an idea of the time scale on which the plasma thermal energy and therefore the plasma pressure can change, ranges from $1 \mathrm{~ms}$ to $100 \mathrm{~ms}$ on TCV. More rapid variations of the plasma energy content are also of great interest, for example those obtained during fast modulation of the heating power aimed at deriving the absorbed power, with a modulation frequency reaching almost $1 \mathrm{kHz}$ [7], or fast MHD events occurring in the plasma such as internal disruptions or ELMs, whose characteristic times are much smaller than $1 \mathrm{~ms}$ [8]. Temporal variations in the plasma diamagnetic flux are intrinsically shielded by image currents in the conducting vacuum vessel, with a characteristic time constant for the TCV vessel of $5.3 \mathrm{~ms}$. Under these conditions an adequate diamagnetic measurement cannot be obtained without carefully compensating for the effect of these currents. 
The following section presents the TCV tokamak experimental set up and the arrangement of the measurement loops that constitute the diamagnetic system. Section 3 is dedicated to an analysis of the signal processing necessary to extract the plasma diamagnetic flux from the measured fluxes with the required specifications. Section 4 describes the electronic hardware that has been developed to realise this signal processing. In section 5 the intricate software compensation to obtain the required absolute flux measurement precision are detailed. Finally, practical situations in which a large bandwidth in the measurement has proven to be valuable are presented. Section 7 concludes.

\section{Experiment hardware}

Figure 1 is a poloidal cross-section of the TCV tokamak. The plasma current induction system is an air core transformer comprising coils A, B, C and D. Flexible shape control requires a large number of poloidal coils and is provided by two stacks of eight coils labelled respectively $\mathrm{E}$ and $\mathrm{F}$. The toroidal field is produced by 16 coils surrounding all the ohmic and shaping coils. To accommodate highly elongated plasmas, the vacuum vessel has an almost rectangular cross-section. It is poloidally and toroidally continuous and is made of stainless steel with thickness varying between $15 \mathrm{~mm}$ and $20 \mathrm{~mm}$, providing a low electrical resistance, necessary for passive stabilisation of highly elongated plasmas whose vertical position is unstable. The drawback is the induction of large vessel currents that must be carefully accounted and compensated for in the diamagnetic measurement.

Also illustrated in figure 1, the diamagnetic measurement system is composed principally of 4 loops. The diamagnetic loop, D, is a single turn loop encircling the vacuum vessel and measuring the total toroidal flux from the toroidal coils, the plasma and the vessel image currents. The compensation loop, $\mathrm{C}$, is an 80 turn coil engraved on a printed board and mounted on the D loop support. The $\mathrm{C}$ loop compensates for the flux from the toroidal coils and its area is adjusted to catch the same flux as the D loop. The A loop is a single turn loop made of mineral insulated coaxial wire and wound directly on the vacuum vessel. It is used to assess the vessel image currents. A fourth loop labelled B, similar to the C loop and mounted on a vertical leg of one of the toroidal coils, is sensitive to the current diffusion in the toroidal coil conductor whose importance is explained later.

On TCV, the plasma-wall separation must remain as low as possible in order that the passive wall stabilisation effect may be used efficiently for plasma vertical position control. The 
tile thickness has thus been fixed at $24 \mathrm{~mm}$, rendering the installation of diamagnetic loops inside the vessel extremely difficult. As a consequence, the double loop method [9][10] cannot be implemented.

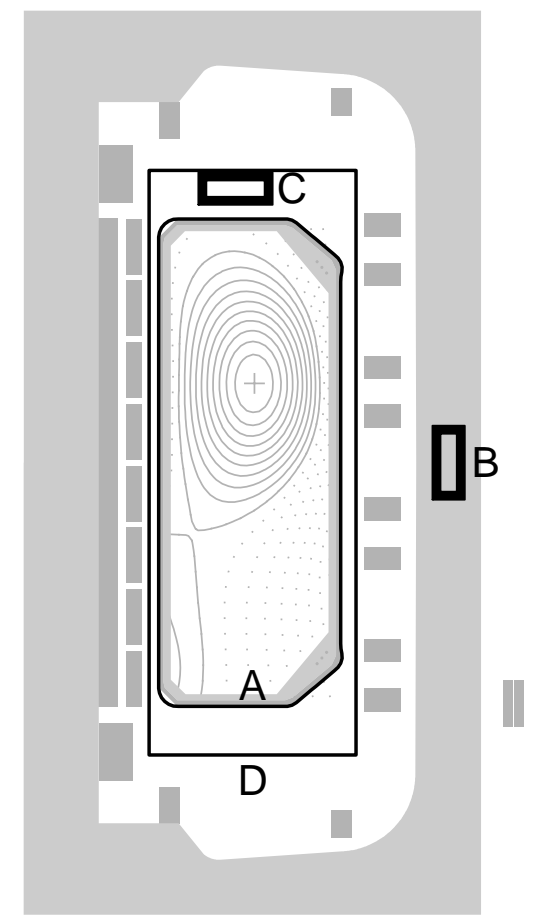

Fig. 1 TCV poloidal cross-section showing the D and A single-turn loops and the $C$ and $B$ multi-turn loops used for the diamagnetic measurements.

\section{Signal processing}

The signal processing of the diamagnetic measurement can be divided in two parts, a analog part and a software part. In the analog part, the signals from the loops are combined, amplified, integrated and filtered. This processing roughly cancels the main toroidal flux sources other than the plasma diamagnetic flux and thus allows all signal levels and bandwidths to match that required for the digitalisation. The software processing cancel the remaining perturbing fluxes. Those arises from imperfections in the analog compensations, from coupling with the poloidal magnetic field and from flux changes due to small loop displacements under mechanical and thermal stresses in the machine. But before selecting an appropriate method for both the analog and the software processing, it is important to formally derive a signal combination and interpretation that can meet the retained objectives in term of measurement precision and bandwidth. This is the goal of this section. 
There are three sources of toroidal magnetic flux: the toroidal flux created by the plasma, $\phi_{p}$, that produced by the current in the toroidal field coils, $I_{t}$, and finally that coming from poloidal current in the conducting vessel, $I_{v}$, induced by mutual inductance with $\phi_{p}$ and $I_{t}$. The fluxes from the plasma and the vessel current are limited inside the vacuum vessel and are not captured by the C loop. Thus voltages measured on each of the D, C and A loop are given by the following equations in the Laplace domain:

$$
\left[\begin{array}{l}
U_{D} \\
U_{C} \\
U_{A}
\end{array}\right]=\left[\begin{array}{lll}
s & s M_{D t} & s M_{D v} \\
0 & s M_{C t} & 0 \\
s & s M_{A t} & s M_{A v}
\end{array}\right]\left[\begin{array}{c}
\phi_{p} \\
I_{t} \\
I_{v}
\end{array}\right]
$$

The mutual inductances between the measuring loops and the poloidal currents are symbolised by the elements of the matrix $M$.

Solving equation (2) for $\phi_{p}$ yields a combination of these three voltages that could be used as an estimate for $\phi_{p}$, the quantity of interest:

$$
\phi_{p}=\frac{M_{A v}}{M_{A v}-M_{D v}} \frac{U_{D}}{s}-\frac{M_{D t} M_{A v}-M_{D v} M_{A t}}{M_{C t}\left(M_{A v}-M_{D v}\right)} \frac{U_{C}}{s}-\frac{M_{D v}}{M_{A v}-M_{D v}} \frac{U_{A}}{s} .
$$

Unfortunately, since the flux from the vessel current seen by loops A and D is the same, $M_{A v}$ and $M_{D v}$ are almost identical and this yields an ill conditioned estimate for $\phi_{p}$.

In the so-called double loop method, this problem does not appear since one or both loops are located inside the vacuum vessel and therefore captures only a fraction of the flux due to the vessel current. In this method, the voltage difference between A and D loops is used, rather than $U_{A}$ itself, giving the following voltage combination:

$$
\phi_{p}=\frac{U_{D}}{s}-\frac{M_{D t} M_{A v}-M_{D v} M_{A t} U_{C}}{M_{C t}\left(M_{A v}-M_{D v}\right)}-\frac{M_{D v}}{M_{A v}-M_{D v}} \frac{U_{A}-U_{D}}{s} .
$$

This has the advantage that no calibration is required for the main loop voltage $U_{D}$ but only adjustment in the compensation gain of the $U_{C}$ and $U_{A}-U_{D}$ terms. In the case of loops located outside the vacuum vessel, or with a single loop, introducing the constraint of the known time 
evolution of the vessel current can alleviate the difficulties of mutual inductance similarity. This evolution is governed by the voltage equation:

$$
0=s \phi_{p}+s M_{v t} I_{t}+s L_{v} I_{v}+R_{v} I_{v}
$$

where $L_{v}$ and $R_{v}$ are respectively the self-inductance and the resistance of the vessel for poloidal currents. Equation (5) can be introduced, for example, by substraction to the third equation of (2):

$$
\left[\begin{array}{l}
U_{D} \\
U_{C} \\
U_{A}
\end{array}\right]=\left[\begin{array}{lll}
s & s M_{D t} & s M_{D v} \\
0 & s M_{C t} & 0 \\
0 & s\left(M_{A t}-M_{v t}\right) & s M_{A v}-s L_{v}-R_{v}
\end{array}\right]\left[\begin{array}{c}
\phi_{p} \\
I_{t} \\
I_{v}
\end{array}\right]
$$

Solving again for $\phi_{p}$ yields the following combination of the measured voltages:

$$
\phi_{p}=\frac{U_{D}}{s}-k_{D} \frac{1+s\left(\tau_{1}+\tau_{2}\right)}{1+s \tau_{1}} \frac{U_{C}}{s}+\frac{\tau_{D v}}{1+s \tau_{1}} U_{A}
$$

where

$$
\begin{gathered}
k_{D}=\frac{M_{D t}}{M_{C t}} \\
\tau_{D v}=\frac{M_{D v}}{R_{v}} \\
\tau_{1}=\frac{L_{v}-M_{A v}}{R_{v}} \\
\tau_{2}=\frac{M_{D v}}{M_{D t}} \frac{M_{A t}-M_{v t}}{R_{v}}
\end{gathered}
$$

In practice, adjusting the various parameters of the voltage combination proposed in equation (7) and given in equations (8) to (11) is too difficult and cannot be achieved with the required precision. Instead, based on the structure of this equation, an approximation must be 
found with acceptable properties for the estimation of $\phi_{p}$ while remaining adjustable using real experiments.

The coefficient $k_{D}$ in the contribution of $U_{C}$ can be adjusted such that in stationary conditions without plasma, in which $I_{v}=0$ and $\phi_{p}=0$, the contribution of the toroidal field coil current $I_{t}$ is cancelled. Inserting these conditions in equation (6) yields the following voltage combination:

$$
\phi_{o}=\frac{U_{D}}{s}-k_{D} \frac{U_{C}}{s}
$$

Due to image currents in the vessel under transient conditions, this combination will display some sensitivity to variation in $\phi_{p}$ and $I_{t}$. This can be expressed by:

$$
\phi_{o}=\phi_{p}+M_{D v} I_{v}=\phi_{p}-M_{D v} \frac{s \phi_{p}+s M_{v t} I_{t}}{R_{v}+s L_{v}}
$$

where $I_{v}$ is deduced from equation (5). The voltage $U_{A}$ may be used to compensate for this sensitivity. The two following equations give the value of $\phi_{o}$ and $U_{A}$ in the absence of plasma $\left(\phi_{p}=0\right)$ :

$$
\begin{gathered}
\phi_{o}\left(\phi_{p}=0\right)=-M_{D v} \frac{s M_{v t}}{R_{v}+s L_{v}} I_{t} \\
U_{A}\left(\phi_{p}=0\right)=\left(s M_{A t}-s M_{A v} \frac{s M_{v t}}{R_{v}+s L_{v}}\right) I_{t}
\end{gathered}
$$

Comparing equation (14) and (15) one deduces:

$$
\phi_{o}\left(\phi_{p}=0\right)=-\frac{\tau_{A}}{1+s \tau_{3}} U_{A}\left(\phi_{p}=0\right)
$$

where

$$
\tau_{A}=\frac{M_{v t}}{M_{A t}} \tau_{D v}
$$




$$
\tau_{3}=\frac{L_{v} M_{A t}-M_{A v} M_{v t}}{R_{v} M_{A t}}
$$

On this basis, it is now possible to define a new voltage combination,

$$
\phi_{1}=\frac{U_{D}}{s}-k_{D} \frac{U_{C}}{s}+\frac{\tau_{A}}{1+s \tau_{3}} U_{A}
$$

that is insensitive to $I_{t}$ at all frequencies and related only to $\phi_{p}$ :

$$
\phi_{1}=\left(1-\frac{M_{D t}}{M_{A t}} \frac{s \tau_{2}}{1+s \tau_{3}}\right) \phi_{p}
$$

It is worth checking whether in this combination the transfer function $1 /\left(1+s \tau_{3}\right)$ in the $U_{A}$ therm can be neglected or not. If yes the simpler combination could be used:

$$
\phi_{2}=\frac{U_{D}}{s}-k_{D} \frac{U_{C}}{s}+\tau_{A} U_{A}
$$

This new combination compared to $\phi_{1}$ suffers from some additional parasitic terms:

$$
\phi_{2}=\phi_{1}+\frac{s \tau_{D v} s \tau_{3}}{1+s \tau_{v}}\left(\frac{M_{v t} l+s \tau_{1}}{M_{A t}} \frac{1+s \tau_{3}}{1+{ }_{p}}+M_{v t} I_{t}\right)
$$

where

$$
\tau_{v}=\frac{L_{v}}{R_{v}}
$$

It is now necessary to examine if these new terms are small enough in the frequency range of interest. In practice, the ratio of mutual inductances can be estimated from the ratio of the areas encircled by the measurement loops. These are:

$$
\begin{aligned}
& A_{v}=0.88 \mathrm{~m}^{2} \\
& A_{A}=0.91 \mathrm{~m}^{2} \\
& A_{D}=1.30 \mathrm{~m}^{2}
\end{aligned}
$$


The vessel characteristic time is extracted from a measurement of the transfer function, shown in figure 2 , between the current in a helical wiring installed inside the vacuum vessel and the D loop. The first cut-off frequency in this transfer function is at $f_{v}=30 \mathrm{~Hz}$ and time constants can be estimated as follows:

$$
\begin{aligned}
& \tau_{v}=\frac{1}{2 \pi f_{v}}=5.3 \mathrm{~ms} \\
& \tau_{D v} \cong \tau_{v} \\
& \tau_{1} \cong 0 \\
& \tau_{2}=\frac{M_{D v}}{M_{D t}} \frac{M_{A t}-M_{v t}}{R_{v}}=\tau_{D v} \frac{M_{A t}}{M_{D t}}\left(1-\frac{M_{v t}}{M_{A t}}\right) \cong 0.023 \tau_{v} \\
& \tau_{3}=\frac{L_{v} M_{A t}-M_{A v} M_{v t}}{R_{v} M_{A t}}=\tau_{v}\left(1-\frac{M_{A v}}{L_{v}} \frac{M_{v t}}{M_{A t}}\right) \cong 0.033 \tau_{v}
\end{aligned}
$$

Introducing these estimations into equation (22) demonstrates that the new parasitic term is clearly dominated by the flux $M_{v t} I_{t}$ which is much larger than $\phi_{p}$ :

$$
\phi_{2}-\phi_{1}=\frac{s \tau_{D v} s \tau_{3}}{1+s \tau_{v}}\left(\frac{M_{v t}}{M_{A t}} \frac{1+s \tau_{1}}{1+s \tau_{3}} \phi_{p}+M_{v t} I_{t}\right) \cong \frac{s \tau_{v}}{1+s \tau_{v}} s \tau_{3} M_{v t} I_{t}
$$

This must, however, remain small compared to $\phi_{p}$, a condition which is satisfied on for frequencies much smaller than

$$
\frac{1}{2 \pi \tau_{3}} \frac{\phi_{p}}{M_{v t} I_{t}}
$$

corresponding to $0.1 \mathrm{~Hz}$, which is far outside the objectives of the measurement. The rectified voltage applied to the toroidal field coils also induces a current ripple of about $10^{-3} I_{t}$ at $1200 \mathrm{~Hz}$. This creates a parasitic component of the order of

$$
s \tau_{3} M_{v t} I_{t} \cong 2 m W b
$$

which also exceeds the expected precision in the measurement. Therefore the transfer function in the $U_{A}$ compensation can not be neglected and equation (19) must be retained. 
Finally it is worth noting that the signal from the D loop is not essential and can be replaced by that of the A loop. In this case, the combination (19) is replaced by

$$
\phi_{1}=\frac{U_{A}}{s}-k_{A} \frac{U_{C}}{s}+\frac{\tau_{A}}{1+s \tau_{3}} U_{A}
$$

with

$$
k_{A}=\frac{M_{A t}}{M_{C t}}
$$

and the relation between this signal and the plasma flux by

$$
\phi_{1}=\left(1-\frac{s \tau_{2}}{1+s \tau_{3}}\right) \phi_{p}
$$

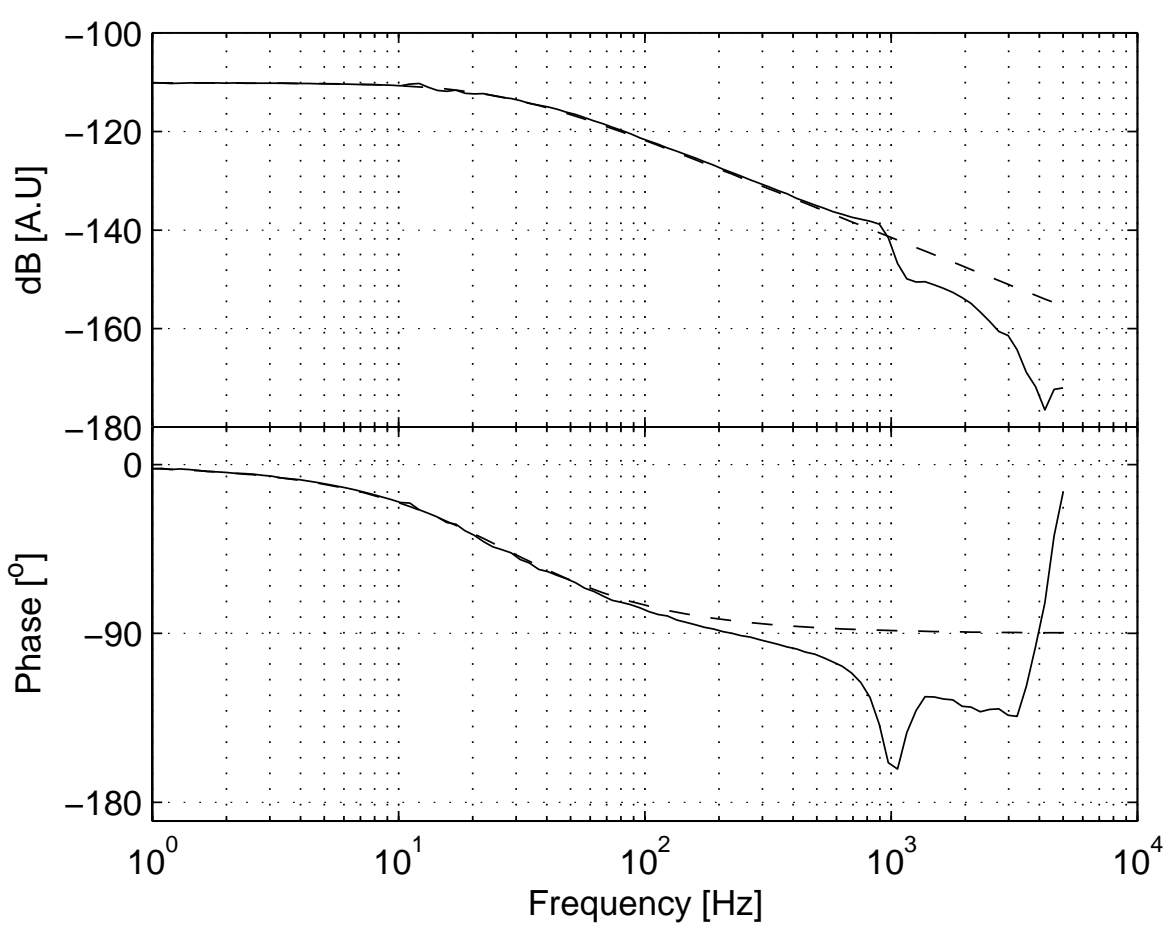

Fig. 2 Transfer function from the flux inside the vessel to the D loop. The dashed line is a fit of a first order transfer function. 


\section{Analog signal processing}

Adequate analog signal processing is necessary before signal digitisation and acquisition because the ratio between the total toroidal flux and that produced by the plasma is much larger than the dynamic range of a common, 12 bit analog to digital converter, preventing digital combination on the full signal range. The signal combination given by equation (29) is therefore implemented in an analog circuit, in the following form:

$$
U_{1}=k_{I}\left(\frac{1}{s \tau_{i}}\left(\frac{1}{2} U_{D}-k_{C} U_{C}\right)+\frac{\tau_{A} / \tau_{i}}{1+s \tau_{3}} U_{A}\right)
$$

where $\tau_{i}$ is the integration time.

Figure 3 gives a block diagram of the hardware used in the TCV diamagnetic diagnostic signal processing. The 3 signals from D, C and A loops are combined in the following steps:

1. The D (or A) and C loop signals are subtracted from each other with a passive resistance bridge, a method guaranteeing high linearity. The $U_{C}$ compensation gain, $k_{C}$, is adjusted during phases where $I_{t}$ is constant, when $U_{A}=0$. A residual level of $0.6 \mathrm{mWb}$ is reached using this analog compensation.

2. The resulting signal is fed into a very low drift integrator whose input stage is a very low noise differential amplifier with a gain of 4 and whose active integration has a time constant of 3 or $12 \mathrm{~ms}$. The overall integration time constant is 1.5 or $6 \mathrm{~ms}$ (including the resistance bridge attenuation) which allows a flux full range of 15 or $60 \mathrm{mWb}$ for an integrator output of $10 \mathrm{~V}$.

3. A loop signal is amplified by a differential band limited amplifier with a gain $\tau_{A} / \tau_{i}$ of 3.74 or 0.94 depending on the selected integrator time constant. The cut-off frequency of the amplifier corresponding to the time constant $\tau_{3}$ is $1270 \mathrm{~Hz}$. It is measured from the phase shift of a $50 \mathrm{~Hz}$ parasitic component in $I_{t}$.

4. The amplifier and integrator outputs are now subtracted at the input of a differential amplifier with a gain of 1,2 or 4 . This allows a flux full scale from 3.75 to $60 \mathrm{mWb}$. The low frequency compensation of $U_{A}$, the coefficient $\tau_{A}$, is tuned using slow ramps in $I_{t}$. A residual flux of $0.05 \mathrm{mWb}$ is achieved.

5. The signals are low-pass filtered with a cut-off frequency of $2.5 \mathrm{kHz}$ and acquired at a sampling rate of $5 \mathrm{kHz}$. 
All loops are referred to the measurement ground. For monitoring purposes the signals of each individual loop are also integrated and amplified, low-pass filtered and acquired with a 12 bit ADC. This signal processing is similar to the other magnetic measurements on TCV and is described in more detail in [11].

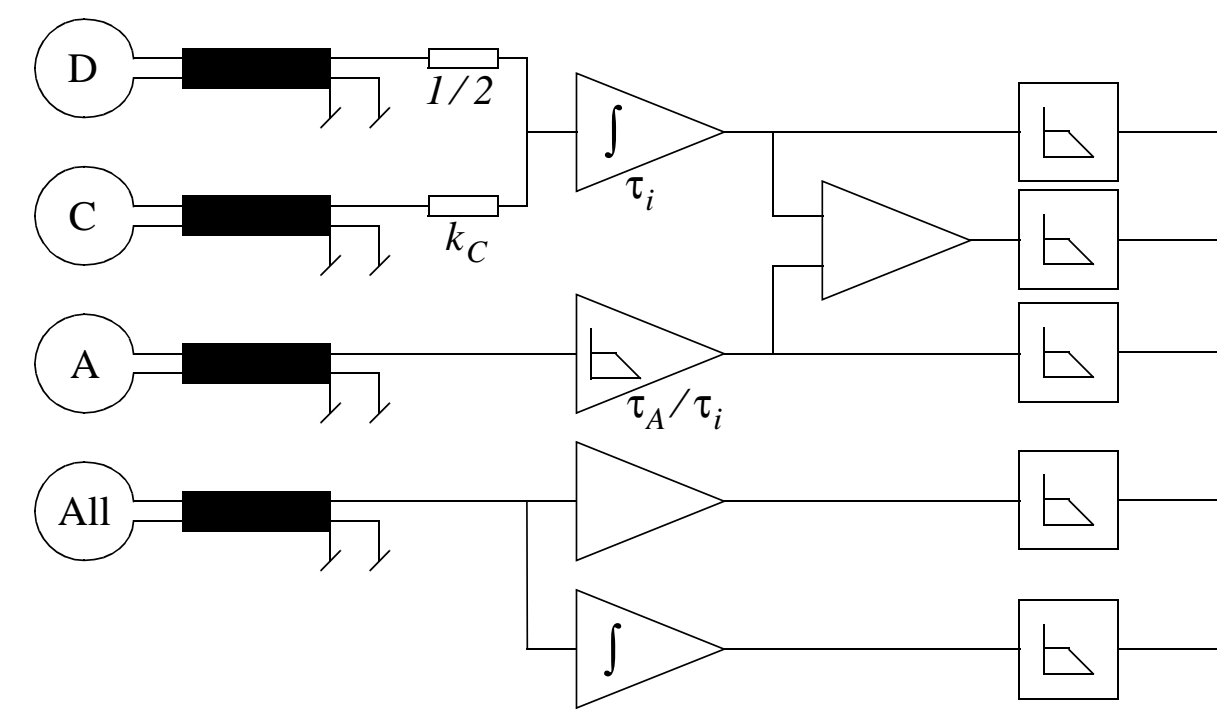

Fig. 3 Block diagram of the analog signal processing.

\section{Software signal processing}

Software processing is used to cancel the remaining parasitic fluxes:

- toroidal coil and vessel eddy current residual fluxes.

- flux coupling from the toroidal currents in the poloidal coils (ohmic transformer, shaping coils, plasma current and vessel current in the toroidal direction).

- flux changes induced by mechanical stress and thermal expansion.

\subsection{Compensation of residual parasitic toroidal fluxes}

Five sources of parasitic toroidal fluxes have been identified, each of which are fine compensated by software signal processing. The coefficients for these compensations are estimated either during phases of the discharge before and after the plasma when there are no currents other than the toroidal coil current and the induced poloidal vessel current or from measurements in the absence of plasma when only the toroidal coils are energised. A combination of such measurements is summarised in figure 4 for several levels of $I_{t}$ and current ramp rates. At a nominal $I_{t}=66 \mathrm{kA}$, the residual flux measured by the analog compensated 
diamagnetic loop is around $1 \mathrm{mWb}$. The value of this residual flux during plateaux in the toroidal coil current, where no vessel current is induced, is plotted in figure 5 as a function of $I_{t}$. This permits the first three compensations to be identified:

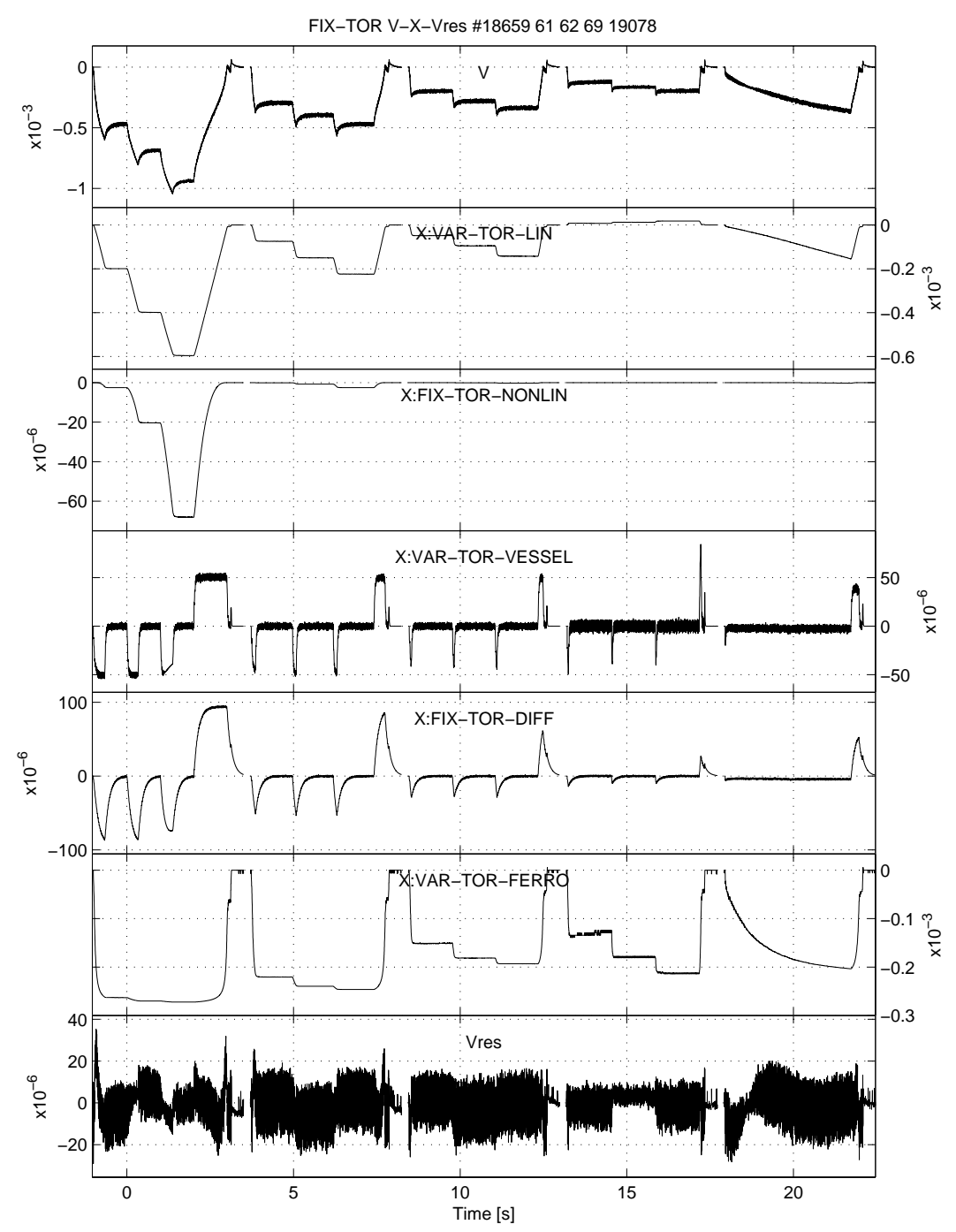

Fig. 4 Residual flux in the analog compensated diamagnetic loop measurement (V) produced by toroidal coil current only, its compensation components (VAR-TROLIN, FIX-TOR-NONLIN, VAR-TOR-VESSEL, FIX-TOR-DIFF, VAR-TOR-FERRO) and the residual flux after software compensation (Vres). Signals are obtained by appending several different pulses in which only the toroidal coils are energised.

VAR-TOR-LIN. A term which is proportional to $I_{t}$; this is a residual component of the direct toroidal flux not fully compensated by analog signal processing and is of the order of $0.6 \mathrm{mWb}$. The integrated signal from loop $\mathrm{C}, U_{C} / s \tau_{C}$ is used to compensate for this term. Its amplitude varies from discharge to discharge because of small displacements in the measurement loops. 
As shown in figure 6, the compensation coefficient is evaluated for each discharge during $I_{t}$ ramp-up and ramp-down phases.

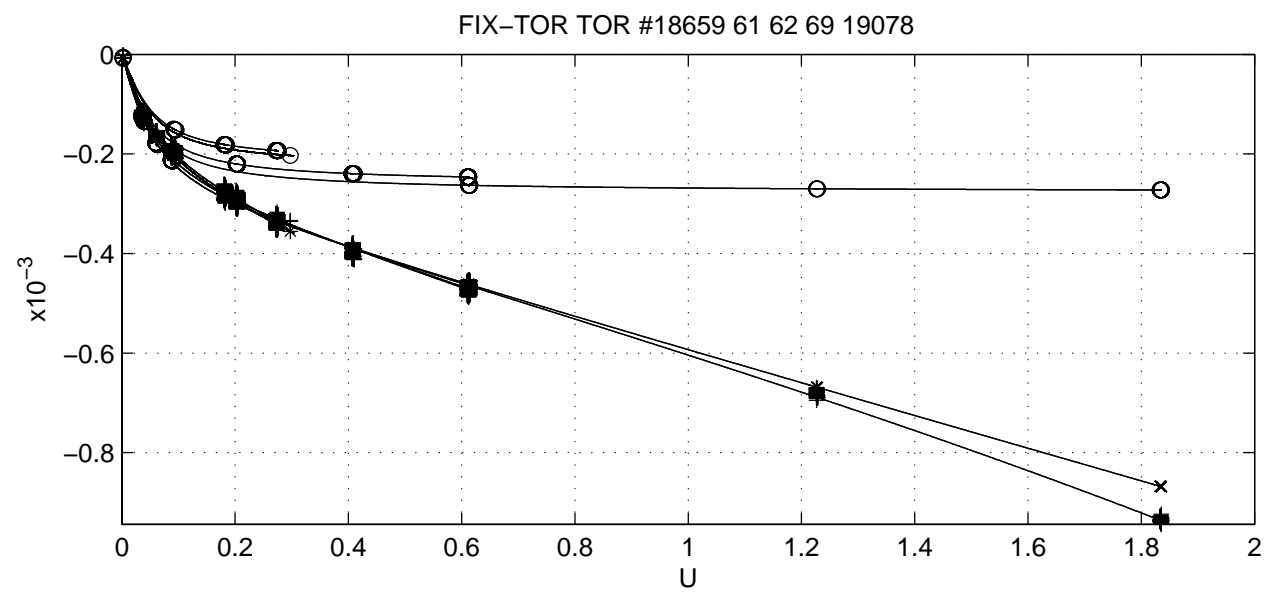

Fig. 5 Residual flux in the analog compensated diamagnetic loop measurement produced by constant toroidal coil current only (+). The cumulative compensations are also plotted: VAR-TOR-FERRO (o); VAR-TOR-FERRO + VAR-TOR-LIN (×) and VARTOR-FERRO + VAR-TOR-LIN + FIX-TOR-NONLIN (*).

FIX-TOR-NONLIN. A departure from the linear dependence can be seen in figure 5 at the highest toroidal coil currents. This is thought to be the consequence of a mechanical deformation of the toroidal coils under the stress from magnetic forces. This deformation is proportional to $I_{t}^{2}$, and gives rise to a flux variation that is proportional to $I_{t}^{3}$. The diamagnetic loop picks up a flux of $0.06 \mathrm{mWb}$ at nominal current. Since this component is rather small, its coefficient is maintained constant and must be carefully deduced during dedicated measurements without plasma, such as those compiled in figure 4.

VAR-TOR-FERRO. Figure 5 clearly shows a background component that saturates at high $I_{t}$. This is due to the presence near the measurement loops, of mechanical components made of ferromagnetic material. The ferromagnetic characteristic is assumed to be of the form $\operatorname{atan}\left(B / B_{c}\right)$, where the critical field, $B_{c}$, is deduced from the measurements without plasma. This characteristic curve is plotted in figure 5. The amplitude of this compensation, which is about $0.5 \mathrm{mWb}$, is derived for each discharge during the ramp up and down of the toroidal coil current, as shown in figure 6 . Because the flux produced by the plasma is small compared to the main toroidal field flux, a mildly ferromagnetic element less that $1 \mathrm{~cm}^{2}$ is sufficient to produce this flux deformation. 
The final necessary compensations linked to the toroidal coil current originate from image currents in both the coils themselves and in the vessel:

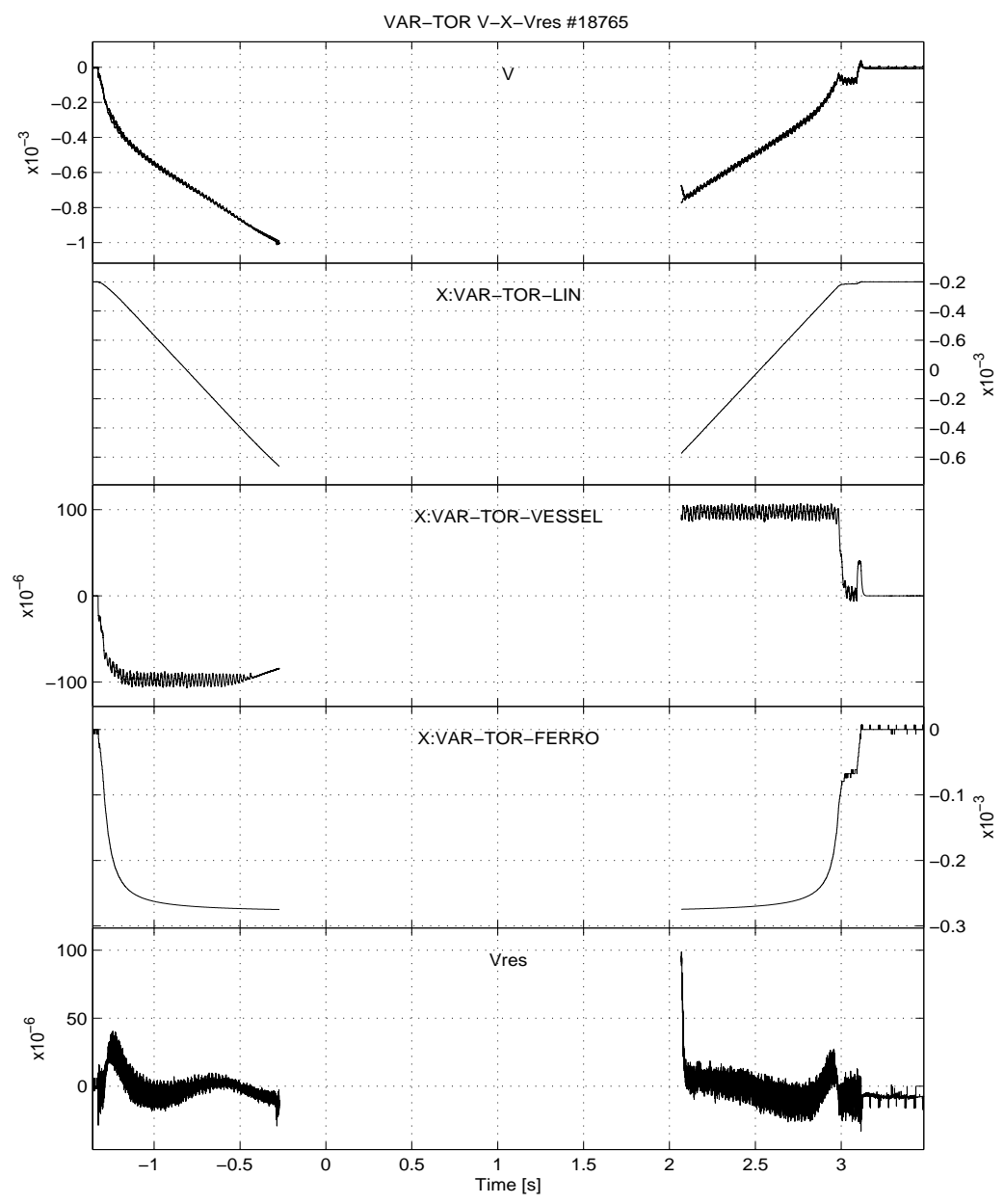

Fig. 6 Residual flux in the analog compensated diamagnetic loop measurement (V) in a real discharge, its compensation components (VAR-TOR-LIN, VAR-TOR-VESSEL, VAR-TOR-FERRO) and the residual flux after software compensation (Vres). Only the phases of the discharge with toroidal coil current only are shown. It is in these intervals that the compensation coefficients are estimated.

VAR-TOR-VESSEL. This is a residual component due to a imperfect analog compensation of the vessel current term, which is $\sim 0.05 \mathrm{mWb}$ during a nominal ramp of $60 \mathrm{kA} / \mathrm{s}$ in the toroidal coil current. The signal used for this software compensation is a digital implementation of the filtered signal $U_{A} /\left(1+s \tau_{3}\right)$. Its amplitude is adjusted during ramp-up and ramp-down phases for each discharge, as shown in figure 6. 
FIX-TOR-DIFF. Figure 4 demonstrates that even when $I_{t}$ has dropped to zero, identifiable where the compensation VAR-TOR-LIN is zero, and when the image current in the vessel has vanished, residual flux persists in the analog compensated diamagnetic signal. This is due to current diffusion in the massive copper toroidal coils which results in a non-uniform current distribution and a corresponding variation in the toroidal flux. This diffusion is inferred using a loop mounted on a vertical leg of one toroidal coil. A fraction of the voltage on this loop is due to the difference in the current density on the inner and outer part of the coil leg due to current diffusion and can be used to compensate for its effect on the toroidal flux. The remaining fraction is directly proportional to the current ramp rate and can be accounted for by using simultaneously the voltage from loop A. Both compensations must therefore be performed together. In a nominal current ramp, this compensation reaches $0.05 \mathrm{mWb}$.

When all five of these compensations are applied, a residual flux $<0.02 \mathrm{mWb}$ is achieved in all practical conditions and a much smaller value when $I_{t}$ is constant. This residual is small enough to guarantee derivation of the plasma pressure using the diamagnetic measurement.

\subsection{Compensation of parasitic poloidal fluxes}

Coupling between the poloidal fluxes and the loops of the diamagnetic system originates mainly from a misalignment of the loops in the poloidal plane. Sources of the poloidal fluxes are the poloidal coils, both ohmic transformer and shaping coils, toroidal image currents in the conducting vessel and the plasma current itself. These couplings are deduced from measurements where each of the 2 ohmic transformer coils and the 16 shaping coils are powered individually without plasma, a summary of which is shown in figure 7 . These couplings are then compensated by digital signal processing for each tokamak discharge.

FIX-POL-LIN. The most direct term in the poloidal flux compensation is simply proportional to the current in the poloidal coils. It is easily measured during periods of constant coil current, as seen in figure 7 . This term may reach $0.4 \mathrm{mWb}$ for individual coil currents but rarely exceeds $0.01 \mathrm{mWb}$ for a real plasma discharge.

FIX-POL-VESSEL. Temporal variations in the poloidal coil currents and in the plasma current value, in its spatial distribution or in the plasma shape and position result in induced toroidal currents in the conducting vessel. To quantify this effect, the first step is to obtain the spatial distribution of this vessel current along the vessel perimeter. This may be accomplished by using the loop voltage on 38 flux loops mounted directly on the vessel walls at evenly spaced 
poloidal positions. Details of the design of these loops may be found in [11]. To compute the spatial distribution of the vessel current, the vessel is divided in 38 segments, each located beneath a flux loop. The voltage equations for both the flux loop voltages, $U_{f}$, and the vessel segments are

$$
\left[\begin{array}{c}
U_{f} \\
0
\end{array}\right]=\left[\begin{array}{ll}
s M_{f a} & s M_{f s} \\
s M_{s a} & R_{s}+s M_{s s}
\end{array}\right]\left[\begin{array}{c}
I_{a} \\
I_{s}
\end{array}\right]
$$

where $I_{a}$ and $I_{s}$ are the poloidal coil and vessel segment currents respectively, $R_{s}$ the electrical resistance of the vessel segments (which have been determined experimentally using the method reported in [11]), and the elements of the matrix $M$ symbolise the mutual inductances. Without recourse to simplification, $I_{s}$ may be derived from (33) by iteration using the relation:

$$
I_{s}=\frac{1}{R_{s}}\left(\left(M_{f a}-M_{s a}\right) s I_{a}+\left(M_{f s}-M_{s s}\right) s I_{s}-U_{f=s}\right)
$$

in which the value of $I_{s}$ used on the rhs is that obtained from the previous iteration. Alternatively, one can use a simplified version of this relation, based on the spatial proximity of flux loops and the vessel segments, implying that the mutual inductances, $M_{f a}$ and $M_{s a}$, as well as $M_{f s}$ and $M_{s s}$, are almost identical. This yields a strait forward estimation of the vessel segment currents requiring no iteration:

$$
I_{s}=-\frac{U_{f=s}}{R_{s}}
$$

A further simplification can be obtained by projecting the spatial distribution of $I_{s}$ onto eigenmodes that are based on an appropriate diagonalisation of the $M_{s s}$ matrix,

$$
R_{s}^{-1 / 2} M_{s s} R_{s}^{-1 / 2}=T_{e s}^{t} \tau_{e} T_{e s}
$$

where the rows of $T_{e s}$ are the eigenmode current distribution and $\tau_{e}$ is the diagonal matrix of the eigenvalues representing the eigenmode characteristic time constant. The latter decrease with increasing mode number so that retaining only the first 15 modes out of the 38 defined by equation (36) is sufficient to cover the bandwidth defined by the sampling period of $0.2 \mathrm{~ms}$. 
Keeping only these modes reduces the number of parameters to derive from a limited number of measurements. The projection used is:

$$
I_{e}=-T_{e s} \frac{U_{f=s}}{R_{s}}
$$

The parasitic poloidal flux due to toroidal vessel currents can reach $0.4 \mathrm{mWb}$ during the plasma breakdown and current ramp phases, when the total vessel current rises up to $100 \mathrm{kA}$ and exceeds the plasma current itself.

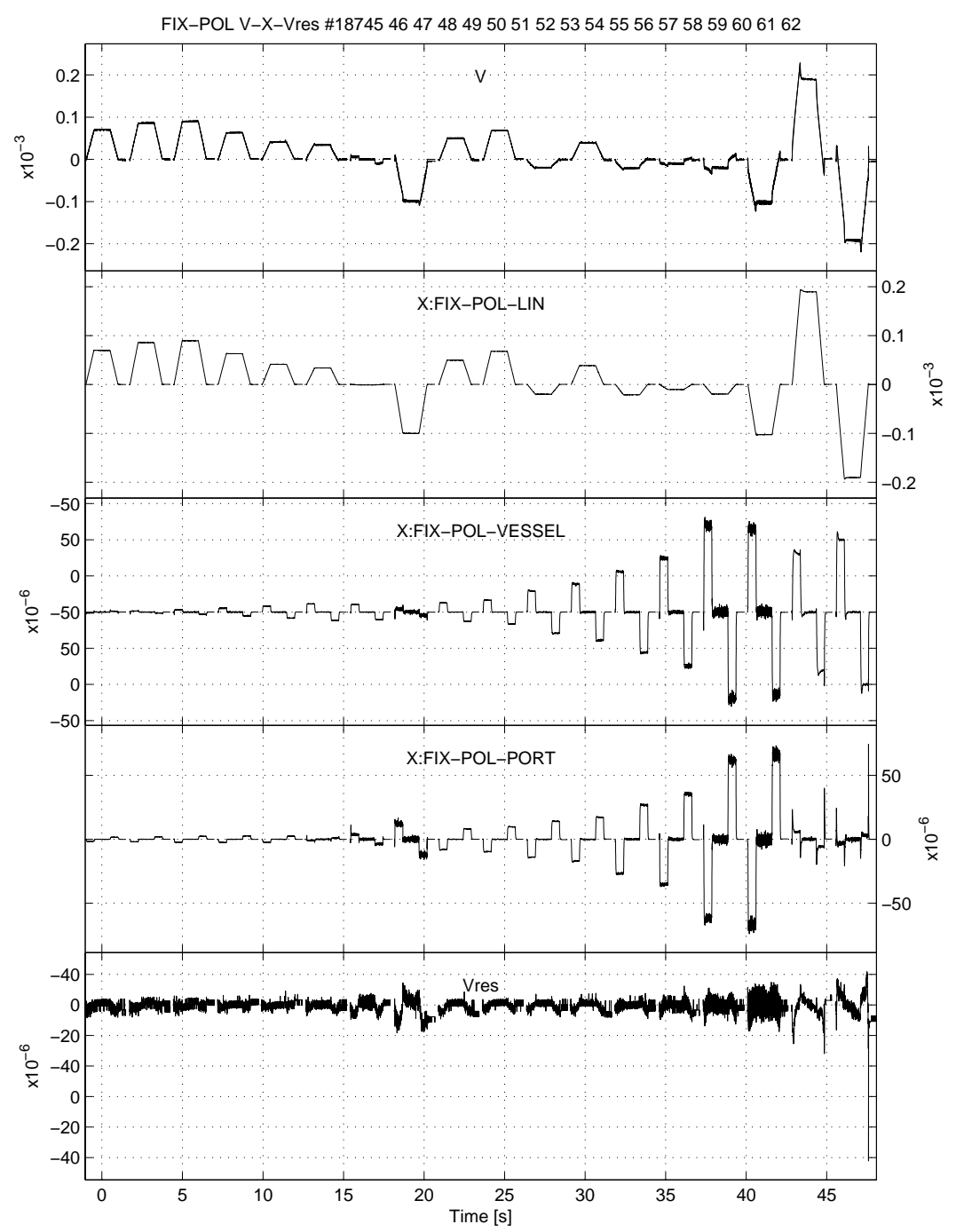

Fig. 7 Flux in the diamagnetic loop (V) produced by poloidal coil currents only, its compensation components (FIX-POL-LIN, FIX-POL-VESSEL, FIX-POL-PORT) and the residual flux after software compensation (Vres). Signals are obtained by appending several different pulses in which coils are individually energised. 
FIX-POL-PORT. The previous compensation deals with toroidally symmetric vessel current. The presence of large ports near the loops of the diamagnetic system creates toroidally localised image currents flowing in the massive metallic structure of these ports. The voltage from the vessel loops is used to obtain the variation in the flux crossing the ports. For example, as sketched in figure 8 , the fraction of the flux crossing the area $A_{f}$ may be approximated by

$$
\frac{A_{f}}{\pi\left(r_{f+1}^{2}-r_{f}^{2}\right)}\left(U_{f+1}-U_{f}\right)
$$

Based on the ratio of the area of the port contained between two adjacent vessel loops and the total area between these two loops, a matrix $T_{q f}$ can be built. The current in the port, $I_{q}$, is then simply this flux variation divided by the electrical resistance of the port structure, $R_{q}$ :

$$
I_{q}=-\frac{T_{q f} U_{f}}{R_{q}}
$$

The parasitic poloidal flux due to image currents in the neighbouring ports can reach $0.2 \mathrm{mWb}$ during plasma current ramp phases.

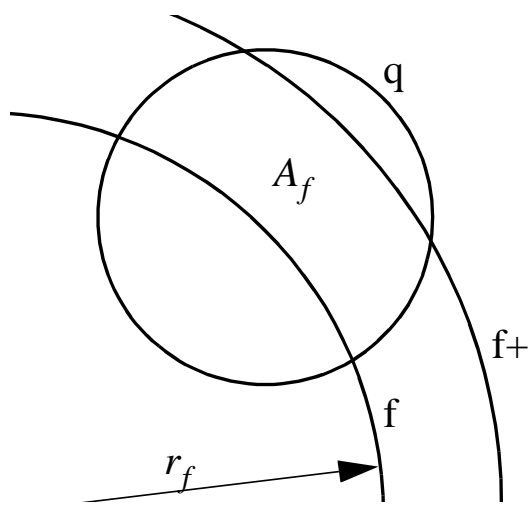

Fig. $8 \quad$ Geometry for estimating port current from vessel loop voltages.

FIX-PLA-LIN. The plasma current itself is a source of poloidal flux that may be captured by misaligned loops. This is rather difficult to measure since it must be separated from the toroidal flux produced by the plasma diamagnetism itself. One common method is to create two plasmas with opposite currents but otherwise similar parameters. The diamagnetic toroidal flux will have the same sign, while the poloidal flux due to the plasma current will change direction. For TCV the situation is more complicated since this parasitic flux certainly depends on the plasma 
position and shape, both of which can and do vary significantly from discharge to discharge. A more general solution would be to determine the loop misalignment and to compute the implied parasitic flux from the plasma current distribution. To this end the D and A loops are assumed to comprise 6 straight, joined segments, the ends of which may be displaced with respect to a perfect poloidal plane, except for one point which is kept fixed, see figure 9. The coil $\mathrm{C}$ is similarly modelled as 4 segments, but these are constrained to rigidly rotate around two orthogonal axes. The resulting 7 displacement parameters are then adjusted to reproduce the coupling between the diamagnetic loop and the 18 poloidal coils, whose geometry and currents are well known. The quality of the adjustment and the misalignment of the two loops are plotted in figure 9. This misalignment is of the order of a few mm for the A and D loops.
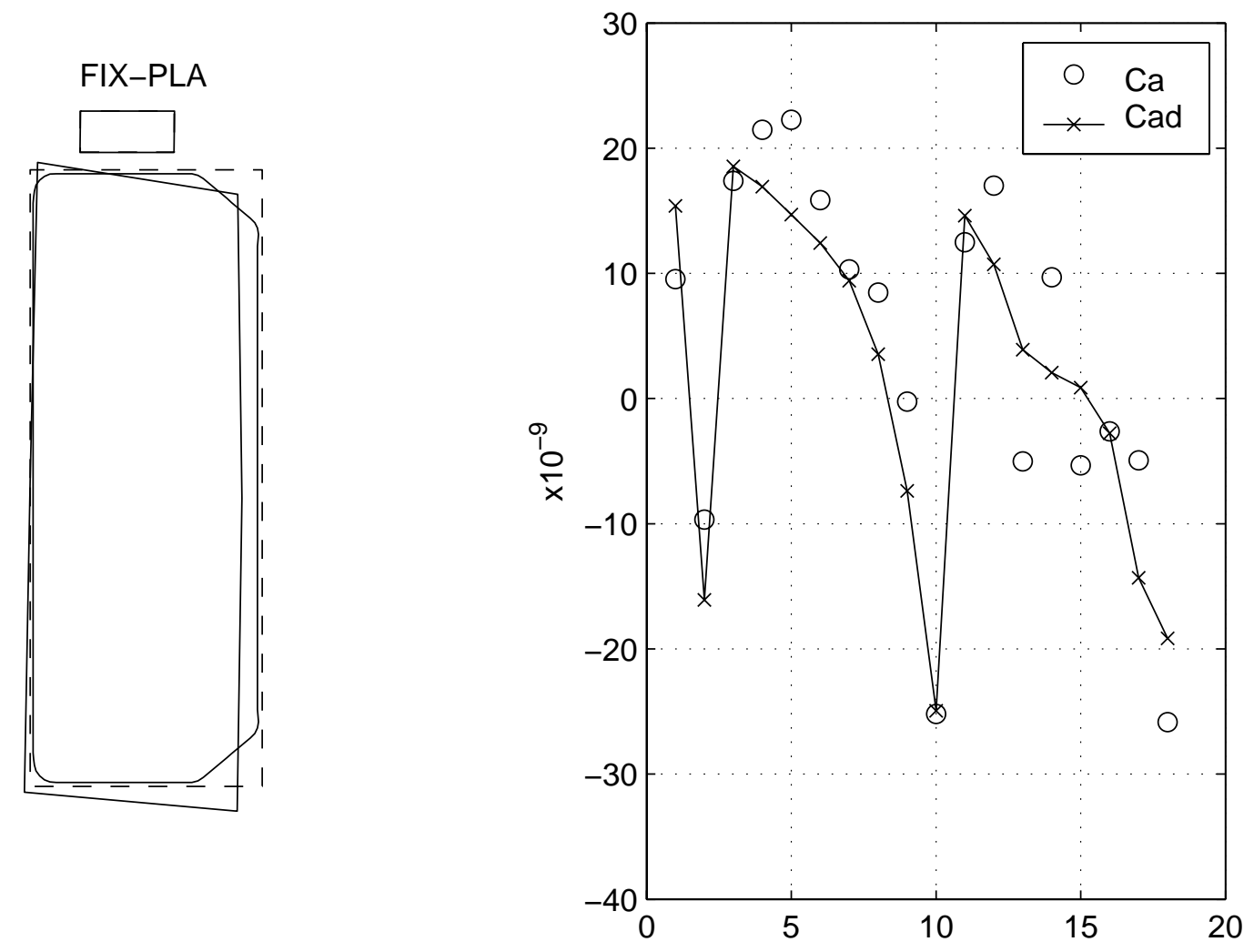

Fig. 9 Left: misalignment of the loops from an ideal poloidal plane, drawing is exaggerated by a factor 10. Right: coupling with the poloidal coils, measured ( $\mathrm{Ca})$ and explained by loop misalignment (Cad).

Once the misalignment is estimated in this way, the mutual inductance between a unit plasma current at any position in the vessel and the misaligned loops can be computed, 
permitting estimation of the coupling with any plasma. The corresponding parasitic flux reaches $0.05 \mathrm{mWb}$ for high plasma current.

Following software compensation for all the five poloidal parasitic fluxes, the residual measured flux is around $0.01 \mathrm{mWb}$. This value is small enough to meet the requirements for the diamagnetic measurement.

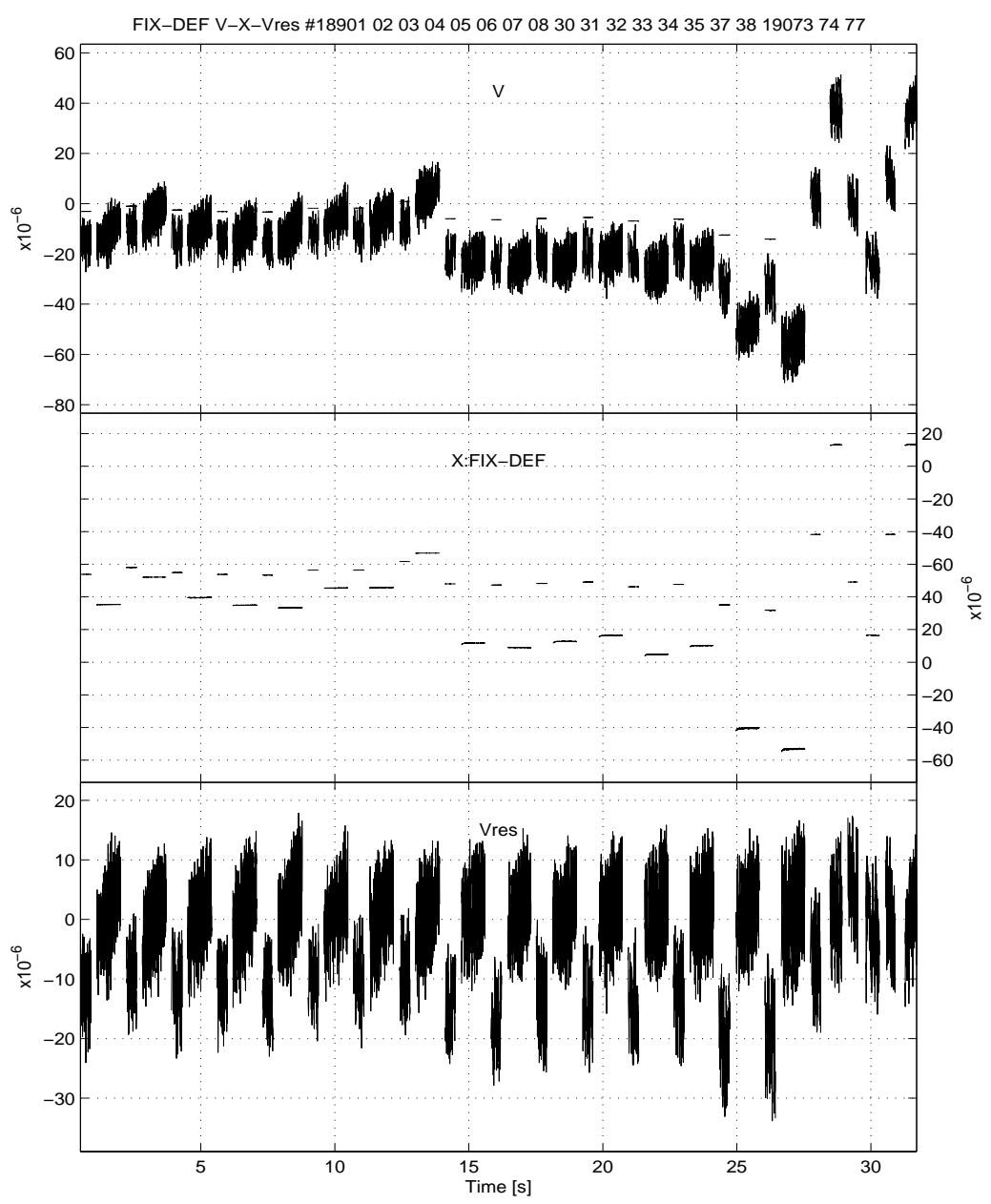

Fig. 10 Flux in the diamagnetic loop (V) produced by poloidal coil currents combined with toroidal coil current, its compensation components (FIX-DEF) and the residual flux after software compensation (Vres). Signals are obtained by appending several measurements.

\subsection{Compensation of deformations}

FIX-DEF. During discharges in which plasma breakdown fails, or with dedicated measurements when the toroidal coils and only one or two poloidal coils are energised, the residual flux after compensation of the toroidal and poloidal parasitic fluxes is observed to 
exceed the compensation level attained when coils are powered separately by values up to $0.06 \mathrm{mWb}$.

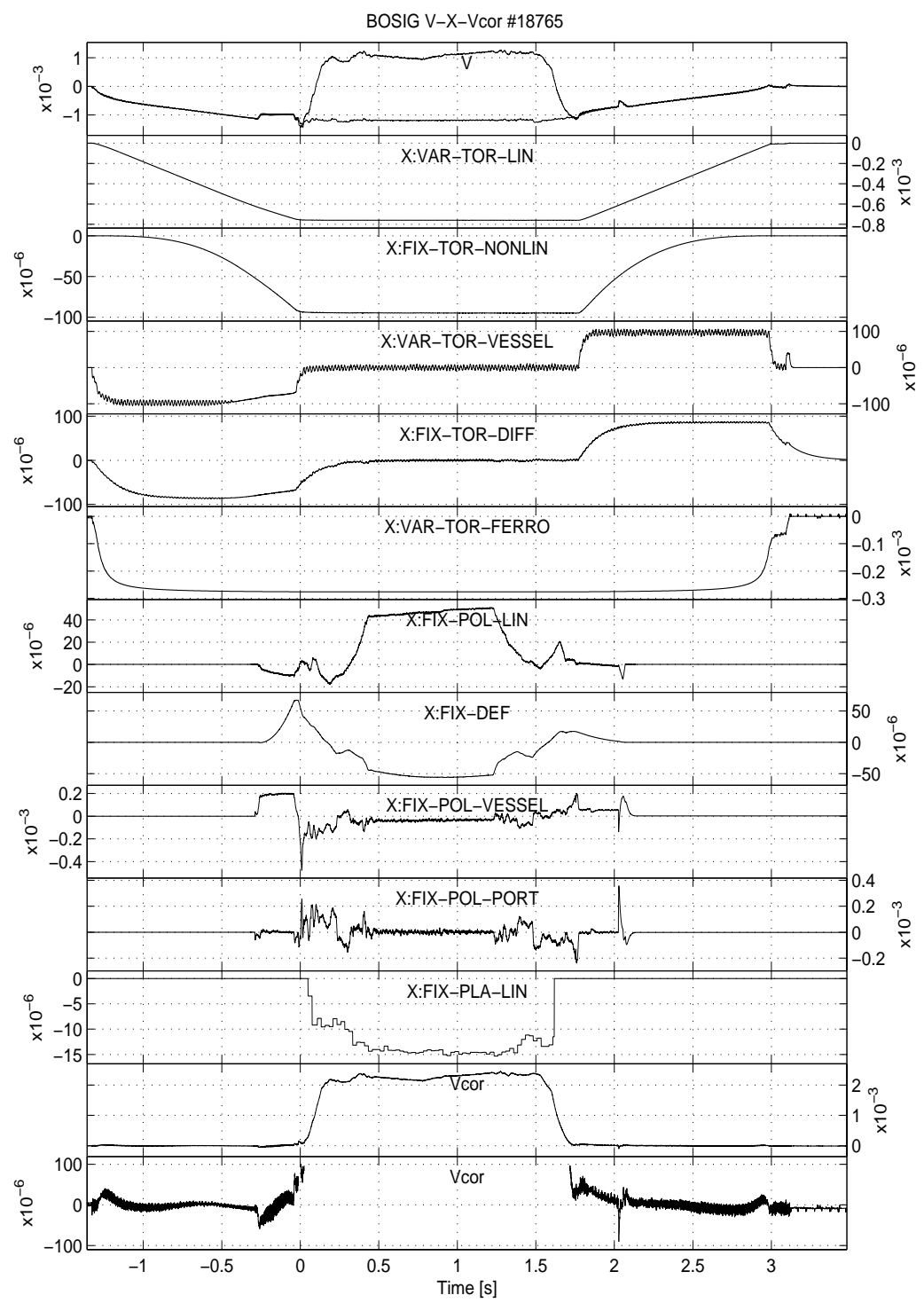

Fig. 11 Analog compensated diamagnetic flux for a real shot $(V)$ with its software compensations $(X)$ and the software compensated diamagnetic flux (Vcor).

Careful inspection of various coil combinations indicates that a possible source of this excess is a non-linear term of the form:

$$
I_{t} I_{a} I_{a^{\prime}}
$$

where $I_{a}$ and $I_{a^{\prime}}$ are the currents in any pair of poloidal coils. This is thought to be the consequence of a deformation of the machine mechanical structure due to the electromagnetic 
force on the coil current $I_{a}$ produced by the magnetic field due to the coil current $I_{a^{\prime}}$. This deformation gives rise to a flux variation proportional to $I_{t}$. Not all poloidal coil pairs have been measured, but the main sources have been identified as the ohmic transformer coils and the selfexpansion of each shaping coil, with a term given by $I_{t} I_{a}^{2}$. As shown in figure 10, correction of this form is sufficient to reduce the residual level below $0.01 \mathrm{mWb}$.

Figure 11 comprises all of the software compensations described above for a real TCV discharge. Inspection of the residual flux before and after the plasma indicates that the required compensation level of $0.04 \mathrm{mWb}$ is achieved.

\section{Applications requiring extended bandwidth}

This section presents two applications in which the large bandwidth of the TCV diamagnetic measurement has proven to be very useful. The first is the measurement of ECH power absorption using modulation of the additional heating power [7]. In order for this power absorption method to succeed, the modulation frequency must be increased up to a point where plasma heat transport processes are ineffective and where the plasma energy content responds adiabatically to the input power. In this situation the modulation in the absorbed power, $\tilde{P}$, is simply related to the amplitude of the modulation in the energy content, $\tilde{W}$, which can be directly derived from the diamagnetic flux:

$$
\tilde{P}=i \omega \tilde{W}
$$

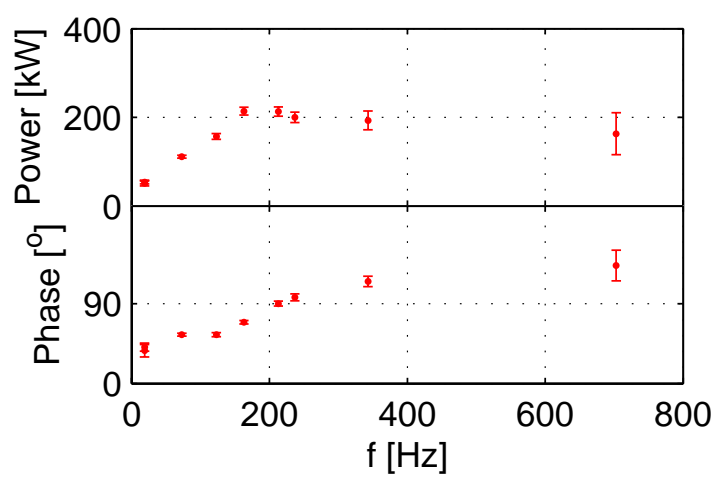

Fig. 12 Estimation of the absorbed power from the diamagnetic flux using power modulation at various frequencies. 
Figure 12 plots the amplitude and phase of $\tilde{P}$, showing that, above a given frequency, the plasma response reaches an asymptotic value satisfying equation (41) and that this relation is experimentally verified up to $700 \mathrm{~Hz}$. This highest modulation frequency is close to the high characteristic frequencies of the diamagnetic measurement and would not have been possible without well tuned compensations of parasitic fluxes and without a correct treatment of the system response at these high frequencies.

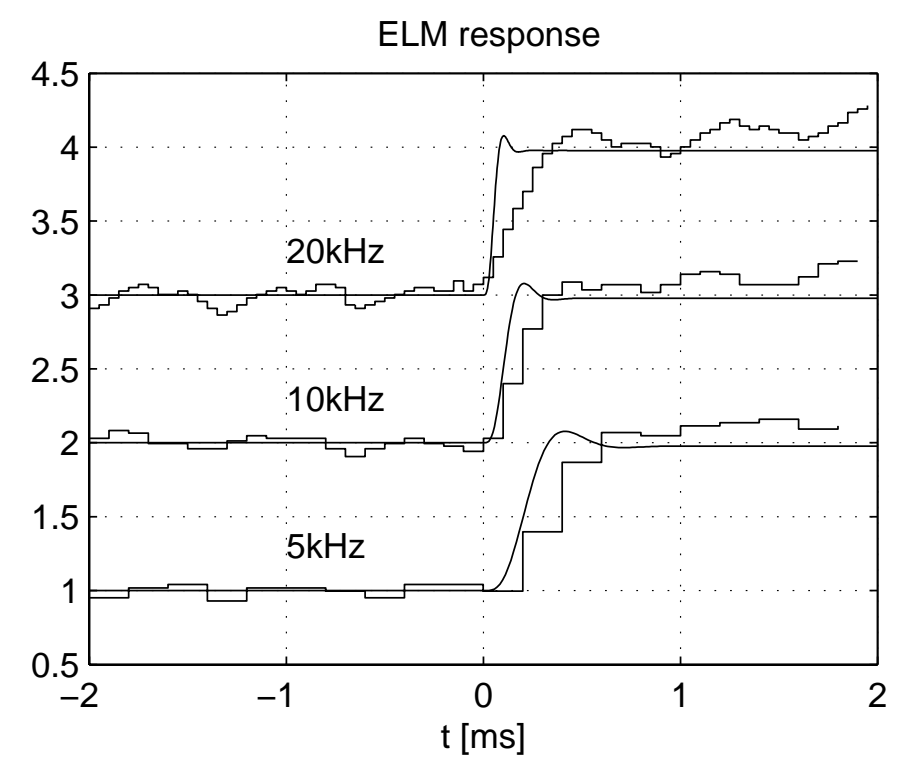

Fig. 13 Diamagnetic flux measurement response (stepped line) to an ELM at various sampling frequencies superposed on the step response (continuous line) of the antialiasing filter set at the Shannon frequency.

A second illustrative application is the response of the measured diamagnetic flux following an ELM. The loss of thermal energy due to an ELM (an MHD instability localised near the plasma edge [12]) can be considered as instantaneous on the time scale of the diamagnetic measurement system. The temporal response has been measured at several sampling frequencies, namely $5 \mathrm{kHz}, 10 \mathrm{kHz}$ and $20 \mathrm{kHz}$, with anti-aliasing filter set at the Shannon limit, half the sampling frequency. The response time of the diamagnetic flux measurement, illustrated in figure 13 , is observed to decrease from the case at $5 \mathrm{kHz}$ to that at $10 \mathrm{kHz}$, but remains unchanged when using $20 \mathrm{kHz}$. This response may be compared to the calculated step response of the anti-aliasing filter, demonstrating that at $5 \mathrm{kHz}$, the time response limitation may be attributed to the low sampling frequency, whilst at $20 \mathrm{kHz}$ the measurement response is slower. In conclusion a bandwidth of around $10 \mathrm{kHz}$ can be achieved. This technique 
has been used to relate in a quantitative way the energy lost from the main plasma during an ELM and that deposited on the target tiles [8].

\section{Conclusion}

On TCV, the constraint that the vertical position of highly elongated plasmas be passively stabilised by the conducting vacuum vessel walls makes the use of an in-vessel diamagnetic flux loop extremely difficult. As a consequence, the so-called double loop technique for compensation cannot be used. Nevertheless, through careful design of analog electronics, the use of compensating loops and software processing, the problems associated with the use of an ex-vessel loop have been overcome.

An appropriate analog processing of the signal measured from a single loop wound outside the vacuum vessel has proven to be adequate for precise compensation of the large image currents induced in the conducting vessel with a bandwidth of $10 \mathrm{kHz}$, large compared to the vessel shielding characteristic time of $5.3 \mathrm{~ms}$. The diamagnetic flux measurement may thus be used to deduce the temporal evolution of the plasma pressure during rapid events, such as additional high frequency modulation of additional heating power or plasma macroscopic instabilities such as ELMs.

Software compensation of several parasitic fluxes must be applied in post-shot analysis to obtain the flux residual level of $0.05 \mathrm{mWb}$ required to accurately estimate the plasma pressure. These parasitic fluxes originate from poloidal eddy currents induced both in the vacuum vessel and in the toroidal field coils themselves. They also arise from coupling between toroidal currents in the poloidal coils, the vessel and the plasma itself and the diamagnetic loops due to small misalignments, deformation of the coils under magnetic forces and the presence of ferromagnetic material near the diamagnetic system loops. The compensations are treated in such a way that the dominant components are easily implemented in the analog control system of TCV [13] with the full bandwidth of the measurement. This allows the signal to be of potential use in real-time control strategies for advanced plasma control.

Acknowledgement. The authors thank the TCV team for their collaboration. This work was partly supported by the Fonds National Suisse de la Recherche Scientifique. 


\section{References}

[1] F. Hofmann, S.C. Jardin, F.B. Marcus et al., Proc. 14th Symp. on Fusion Technology, Avignon (1986) 687.

[2] F. Hofmann, J.B. Lister, M. Anton et al., Plasma Phys. Contr. Fusion 36 (1994) B277.

[3] S. Alberti et al., Fusion Eng. Design 53 (2001) 387.

[4] T.P. Goodman et al., Proc. 19th Symp. on Fusion Technology, Lisbon (1996) 565.

[5] J.-M. Moret et al., Plasma Physics and Controlled Fusion 44 (2002) B85.

[6] K. Miyamoto, Plasma Physics for Nuclear Fusion, MIT Press, Cambridge, 1980.

[7] A. Manini, J.-M. Moret, S. Alberti et al., Plasma Physics and Controlled Fusion 44 (2002) 139.

[8] R.A. Pitts et al., "ELM driven divertor target currents on TCV", submitted for publication in Nuclear Fusion.

[9] T. Mizuuchi et al., Review of Scientific Instruments 72 (2001) 3859.

[10] E. Joffrin and P. Defrasne, Review of Scientific Instruments 73 (2002) 2266.

[11] J.-M. Moret et al., Review of Scientific Instruments 69 (1998) 2333.

[12] H. Zohm, Plasma Physics and Controlled Fusion 38 (1996) 1213.

[13] J.B. Lister, F. Hofmann, J.-M. Moret et al., Fusion Technology 32 (1997) 321. 